\title{
An Efficient Scheduling Algorithm for Cluster in Wireless Sensor Networks MAC Protocol ${ }^{*}$
}

\author{
Yifan Zhao, Liyong Bao, Shaowen Yao \\ School of Information Engineering, Yunnan University, Yunnan Province, China \\ lybao@ynu.edu.cn,bly.yx@163.com
}

\begin{abstract}
As one of the most important techniques in the 21st century, Wireless Sensor Networks(WSNs) receive a universal attention to the research on its key techniques. A solution is urgently expected to meet the higher-quality service requirements of new applications on the limited bandwidth of wireless networks. This paper proposes a scheduling Algorithm fit for the MAC scheme of WSNs, which has made possible the polling service capable of differentiating services of the cluster head node of two priority levels. The theoretical model of this scheme is established through Markov chain and probability generating function. Mathematical analysis is made on the mean queue length, the mean inquiry cyclic time. It turns out that the findings from theoretical analysis correspond well with those from simulated experiments.
\end{abstract}

Index Terms - WSNs, MAC scheme, priority-based, mean queue length, mean cyclic time.

\section{Introduction}

Tensing technology are entering a new era of wireless sensor network[1]. The tiny sensing nodes, which consist of sensing, data processing and wireless communication components in WSNs, are deployed densely and randomly in large numbers[2]. The tiny nodes form the network in a selforganization manner. The sensing performance and reliability have been improved significantly in WSNs.

In many WSNs applications, the data gathering has the Quality of Services(QoS) requirements in terms of BER performance, end-to-end timeliness and reliability, etc. However, since the sensor nodes are battery driven and it is impractical to recharge the battery for so many nodes after deployment, energy efficiency has been a key concern in the research work of WSNs[3-7]. Therefore, to design the scheme with low energy consumption and flexible QoS provisioning is the guarantee for the efficient information gathering in WSNs[8]. Wireless transmission technologies have been identified as the key technologies determining the energy consumption and QoS, which are also the basis for other technologies[9]. Generally, distance between Cluster head and other cluster head is farther, as illustrated in the Fig.1. Wireless transmission between the different clusters also need more power of radio. Therefore, the high-efficient communication between the different clusters appropriate for low energy consumption system remains the key and hot issue in WSNs researches.

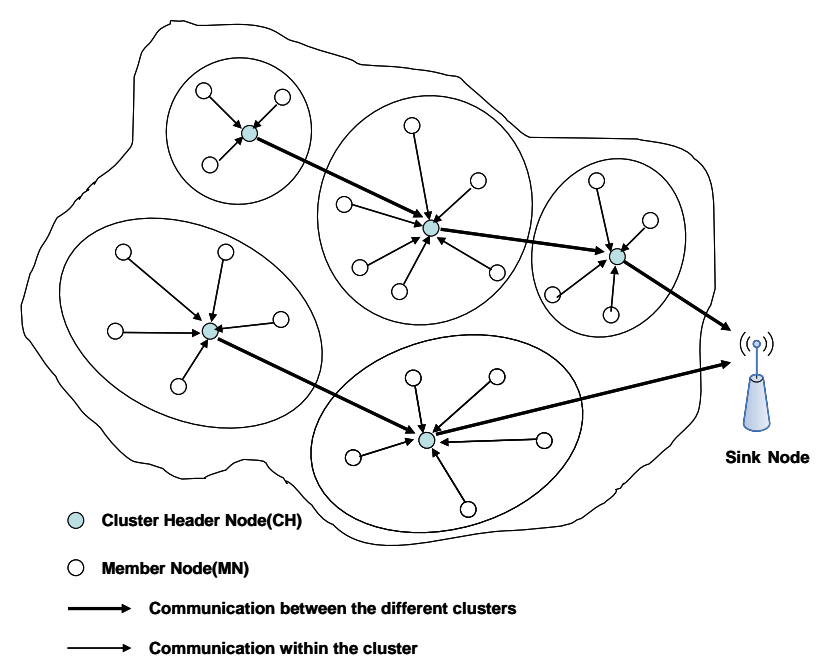

Fig. 1 WSNs network topology structure model

As we all know, the quality of WSNs service mechanism determines the quality of network service[10]. Quality of Service (QoS) is always the basic and hot issue in WSNs[11]. Medium Access Control (MAC) protocol is an important part in the network protocol stack of WSNs, and it is mainly used to coordinate nodes to access the shared wireless channel.

This dissertation proposes an algorithm for polling scheme based on clustering. In the suggested algorithm Cluster Header $(\mathrm{CH})$ polls the active nodes in a polling table which is used to register node addresses and priorities in a cluster to guarantee a QoS scheme of differentiated traffic priorities. The polling scheme not only avoids collisions but also reduces energy consumption for the only services to active nodes[4]. Furthermore, priority-based services can save energy to a large extent. Therefore, in this thesis, the author carried out a deep and systematic research work on the energy efficiency and QoS guaranteeing by establishing Cluster-based MAC Scheme in WSNs.

The system is made up of one Cluster Head(CH), one key node $h$, and $N$ common nodes. The higher-priority key node applies an exhaustive-service policy with less time delay, which fits for the communication between the cluster and its upstream cluster head node. Meanwhile, the lower-priority common nodes use the gated policy to facilitate data transmissions within the cluster. The higher and the lower

\footnotetext{
* This work is supported by the Nation Natural Science Foundation of China (No.61072079).
} 
nodes receive alternatively the polling connection service from $\mathrm{CH}$.

\section{The Theoretical Model of the Scheme}

A. The Operational Mechanism and Variable Definition of the System Model

Polling services at each node include the following processes:

When the arrival process meets with the condition of Poisson, the information packets entering each common node follow the independent and identical distribution of probability, with mean value respectively as $\lambda_{i}(i=1,2, \cdots, N)$.

The information packets entering key node follow the same independent and identical distribution of probability, with mean value respectively as $p \lambda_{h}, 0 \leq p \leq 1$. The $\mathrm{CH}$ polls at the $\boldsymbol{i}(i=1,2, \cdots, N)$ common node at the time of $t_{n}$, with the information packets waiting to be transmitted in the $i$ node is $\xi_{i}(n)$, and the system status variable being $\left\{\xi_{1}(n), \xi_{2}(n), \cdots, \xi_{N}(n), \xi_{h}(n)\right\}$. The CH provides transmission service for the $\boldsymbol{i}$ common node under the limited ( $k=1$ ) service policy. After its service for the $i$ node, the server starts to poll at the key node via a transfer time of $u_{i}(n)$, with the information packets entering the $j$ node $(j=1,2, \cdots, N, h)$ at the time of $u_{i}(n)$ is $\mu_{j}\left(u_{i}\right)$. Then, the $\mathrm{CH}$ inquires the information packets queuing for transmission in the memory of the key node at the time of $t_{n}^{*}$. At this moment, the number of information packets queuing for transmission in the memory of the key node is $\xi_{h}\left(n^{*}\right)$ and the system status variable is $\left\{\xi_{1}\left(n^{*}\right), \xi_{2}\left(n^{*}\right), \cdots, \xi_{N}\left(n^{*}\right), \xi_{h}\left(n^{*}\right)\right\}$. If the memory of the key node is not blank, the transmission of information packets is completed via exhaustive service rules. When the memory of the central station is blank, the $\mathrm{CH}$ starts the transmission service for the information packets in the $i+1$ common node at the time of $t_{n+1}$. At this moment, the system variable is $\left\{\xi_{1}(n+1), \xi_{2}(n+1), \cdots, \xi_{N}(n+1), \xi_{h}(n+1)\right\}$.

\section{B. The Operational Conditions for the System}

According to the operational process of the system, the operational conditions are defined as follows:

1) The time of information packets transmission in the memory the common node follows an independent and identically distributed probability, with its $L S T$ is $\tilde{B}_{i}\left(s_{i}\right)$, and the second-order origin moment respectively is $-\tilde{B}_{i}^{\prime}(0)=\beta$ and $\tilde{B}^{\prime \prime}(0)=\tilde{B}^{\prime \prime}(0)$.

2) The time of information packets transmission in the memory the key node follows an independent and identically distributed probability, with its $L S T$ is $\tilde{B}_{h}\left(s_{h}\right)$, and the mean and the second-order origin moment respectively is $-\tilde{B}_{h}^{\prime}(0)=\beta_{h}$ and $\tilde{B}_{h}^{\prime \prime}(0)$.

3) The transfer time when the $\mathrm{CH}$ polls from the $i$ node to the key node follows an independent and identically distributed probability, with its $L S T$ being $\tilde{R}_{i}\left(s_{i}\right)$, and the second-order origin moment respectively is $-\tilde{R}_{i}^{\prime}(0)=\gamma$ and $\tilde{R}_{i}^{\prime \prime}(0)=\tilde{R}^{\prime \prime}(0)$.

4) The static variable of the time for the exhaustive service of information grouping arriving at the key node follows an independent and identical distribution of probability, with the distributional probability generating function as $H_{h}(0)$.

5) The $\mathrm{CH}$ serves the information packets in the memory of each station based on the FCFS rule.

\section{Probability Generating Function of the System Status Variable}

When the $\mathrm{CH}$ serves the $i+1$ station at the time of $t_{n+1}$, the equation goes as:

$$
\left\{\begin{array}{l}
\xi_{h}(n+1)=0 \\
\xi_{i}\left(n^{*}\right)=\xi_{i}(n)+\mu_{i}\left(u_{i}\right)+\eta_{i}\left(v_{i}\right)-1 \quad i \neq j \\
\xi_{j}(n+1)=\xi_{j}\left(n^{*}\right)+\eta_{j}\left(v_{h}\right) \\
\quad j=1,2, \cdots, N, h
\end{array}\right.
$$

In the equation, $v_{h}(n)$ refers to the time of the CH's transmission service for the information packets at the key node, while $\eta_{j}\left(v_{h}\right)$, the information packets entering the memory of the $j$ node at the time of $v_{h}$.

Based on the above operational process, the status of the polling system can be described by Markov Chain which is noncyclic and ergodic. Stable state is there on condition of $\sum_{i=1}^{N} \rho_{i}+\rho_{h}=N \rho+\rho_{h}<1$,with $\rho_{i}=\lambda_{i} \beta_{i}, \rho_{h}=p \lambda_{h} \beta_{h}, i=1,2, \cdots, N$.

When the $\mathrm{CH}$ polls at the key node at the time of $t_{n}^{*}$, the probability generating function of system status is illustrated as:

$$
\begin{aligned}
& \tilde{G}_{i h}\left(z_{1}, z_{2}, \cdots, z_{N}, z_{h}\right)=\lim _{t \rightarrow \infty} E\left[\prod_{i=1}^{N} z_{i}{ }^{\xi_{i}\left(n^{*}\right)} z_{h}{ }^{\xi_{h}}\left(n^{*}\right)\right] \\
& =\tilde{R}_{i}\left(\sum_{k=1}^{N} \lambda_{k}\left(1-z_{k}\right)+\lambda_{h}\left(1-z_{h}\right)\right)\left\{\frac{1}{z_{i}} \tilde{B}_{i}\left(\sum_{j=1}^{N} \lambda_{j}\left(1-z_{j}\right)+\lambda_{h}\left(1-z_{h}\right)\right) \bullet\right. \\
& {\left[\tilde{G}_{i}\left(z_{1}, z_{2}, \cdots, z_{N}, z_{h}\right)-\left.\tilde{G}_{i}\left(z_{1}, z_{2}, \cdots, z_{N}, z_{h}\right)\right|_{z_{i}=0}\right]} \\
& \left.+\left.\tilde{G}_{i}\left(z_{1}, z_{2}, \cdots, z_{N}, z_{h}\right)\right|_{z_{i}=0}\right\}
\end{aligned}
$$

When the server polls at the $i+1$ station at the time of $t_{n+1}$, the probability generating function of system status is: 


$$
\begin{aligned}
& \tilde{G}_{i+1}\left(z_{1}, z_{2}, \cdots, z_{N}, z_{h}\right)=\lim _{t \rightarrow \infty} E\left[\prod_{i=1}^{N} z_{i}^{\xi_{i}^{(n+1)}} z_{h}^{\xi_{h}^{(n+1)}}\right] \\
& =\tilde{G}_{i h}\left(z_{1}, \cdots, z_{N}, \tilde{H}_{h}\left(\sum_{k=1}^{N} \lambda_{k}\left(1-z_{k}\right)\right)\right)
\end{aligned}
$$

With $\tilde{H}_{h}(s)=\tilde{B}\left(s+\lambda_{h}\left(1-\tilde{H}_{h}(s)\right)\right)$

\section{The Analysis of Mean Queue Length}

Given that $g_{i}(j)$ is the average number of information packets number at the $j$ node buffer when the $i$ node starts service at the time of $t_{n}$, then

$$
\begin{gathered}
g_{i}(j)=\lim _{z_{1}, \cdots, z_{N}, z_{h} \rightarrow 1} \frac{\partial G_{i}\left(z_{1}, z_{2}, \cdots, z_{N}, z_{h}\right)}{\partial z_{j}} \\
i=1,2, \cdots N, h ; \quad j=1,2, \cdots N, h
\end{gathered}
$$

When the $\mathrm{CH}$ starts its service for the $i$ node, the average information packets stored in the memory of the $i$ node are $\tilde{g}_{i}(i)$. Then, the mean queuing length of the common nodes can be calculated with the first-order local derivation via (1) and (2) equations. Under the condition of the symmetrical parameters, $\tilde{g}_{i}(i)$ can be derived as the following via above equations:

$$
\begin{aligned}
& \tilde{g}_{i}(i)=\lim _{z^{\cdots} z_{z^{\prime}} z^{\rightarrow 1}} \frac{\partial G_{i}\left(z_{1}, z_{2}, \cdots, z_{N}, z_{h}\right)}{\partial z_{i}} \\
& =\frac{N r \lambda^{2}}{2\left(1-\rho_{h}\right)\left(1-\rho_{h}-N \rho\right)\left(1-\rho_{h}-N \lambda(\gamma+\beta)\right.} \bullet \\
& \left\{\left(1-\rho_{h}-N \rho\right) \frac{\tilde{R}^{\prime \prime}(0)}{r}+\lambda_{h} \tilde{B}^{\prime \prime}(0)+N \lambda \tilde{B}^{\prime \prime}(0)\right. \\
& +2 N r \rho-(N-3) r \rho_{h}-(N+1) \gamma \\
& \left.+2\left(1-\rho_{h}\right)\left(1-\rho_{h}-N \rho\right) / \lambda\right\}
\end{aligned}
$$

When the $\mathrm{CH}$ polls at the key node, the average information packets stored in the memory of the key node are $\tilde{g}_{i h}(h)$. Likewise, the mean queuing length of the key node can be calculated in the same way:

$$
\begin{aligned}
& \tilde{g}_{i h}(h)==\lim _{z_{1} \cdots, z^{*} z_{h} \rightarrow 1} \frac{\partial G_{h}\left(z_{1}, z_{2}, \cdots, z_{N}, z_{h}\right)}{\partial z_{h}} \\
& =p \lambda_{h}\left(r_{i}+\frac{\rho_{i} \sum r_{k}}{1-\rho_{h}-\sum \rho_{k}}\right)
\end{aligned}
$$

\section{E. The Analysis of the Mean Inquiry Cycle}

Based on the operational mechanism of the system and the theory of queuing, the mean inquiry cycle can be derived as:

$$
E\left[\theta_{i}\right]=\frac{\sum r_{k}}{1-\rho_{h}-\sum \rho_{k}}
$$

\section{Theoretical Calculation, Simulated Experiment and Analysis}

Both theoretical and simulated models are set in identical symmetrical conditions under which each node's information packets arrival follows the Poisson distribution with $\lambda$ as the parameter, and all the node parameters follow the distribution of identical laws. The time slot width is taken as $15 \mu \mathrm{s}$, with the length of information packets being 8100bits. With $N=9, \beta_{h}=\beta_{i}=10, \lambda_{h}=\lambda_{i}=\lambda, \gamma=1$, the system meets the stable condition of $\sum_{i=1}^{N} \rho_{i}+\rho_{h}<1$. The data in the following charts show that the theoretical value of the system model in this research correspond well with that of the experiment and it can represent the first order feature of the system.

1) Considering the system model and operation mechanism, System shows that the key node gets $2 N$ times exhaustive-service in a polling cycle, so that access channel opportunities and transmission quality get more priority guarantee.

2) Fig. 2 reveal the tendency that the mean queuing length of both key and common nodes changes along with the variations of the number of system nodes and arrival rate, which in turn shows that the mean queuing length of the key node has been clearly differentiated from that of the common nodes. With the increase of the common node number $(N)$ and the arrival rate $(\lambda)$, the mean queuing length of the key node is much smaller than that of the common nodes. In other words, the central node enjoys a smaller mean queuing length.

3) Fig.3 reveal that each node in the system enjoys identical mean cycle period and that the system's mean inquiry cycle is greatly influenced by the number of nodes since the average inquiry cycle increases rapidly along with the increase of the number of nodes.

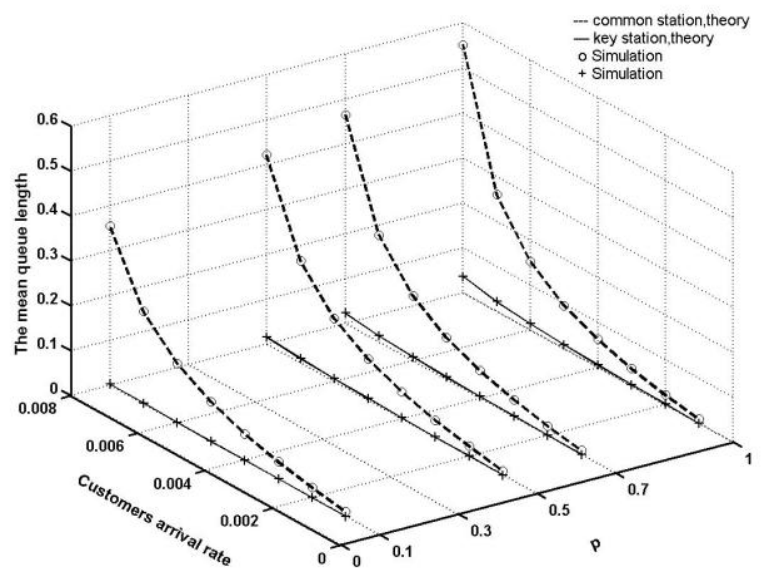

Fig. 2 The mean queue length 


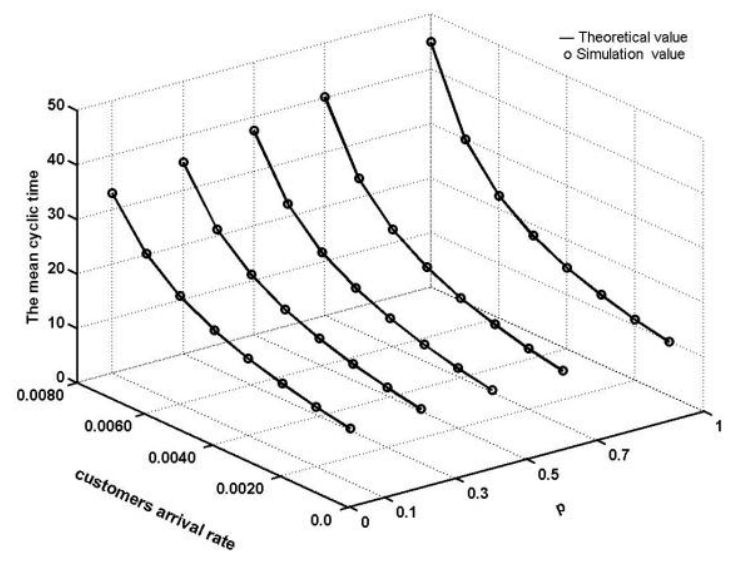

Fig. 3 The mean cyclic time of the system

\section{Conclusion}

In this paper, a research work on MAC scheme in WSNs has been done with focus on energy efficiency and QoS guaranteeing. This efficient scheme embodies the advantages of exhaustive and limited policy service, and it classifies the system nodes by priority at two levels to enhance the fairness and flexibility of the service system. The higher-priority key node applies an exhaustive-service policy with more opportunities for routing so that transmission quality between the different clusters gets better guaranteed. The findings of theoretical calculation correspond well with those of simulated experiments. The theoretical and simulated findings all reveal that system performance can achieve the optimization effect. It concludes that this scheme enables WSNs to take on the features of good utility, good function.

\section{Acknowledgment}

Our sincere thanks to Professor Zha Guangming of UESTC! We will always cherish the memory of Professor Zhao Dongfeng of Yunnan University. The authors would like to give their sincere thanks to the financial support by the Nation Natural Science Foundation of China (No.61072079).

\section{References}

[1] Demirkol I, Ersoy C, Alagoz F, "MAC protocols for wireless sensor networks: a survey," IEEE Communications, vol. 44, pp. 115-121,2006.

[2] Akyildz I F, Melodia T, Chowdhury K R, "A survey on wireless multimedia sensor networks," The International Journal of Computer and Telecommunications Networking, vol.51, pp.921-960, 2007.

[3] Lin Xiaohui, Yukwong Kwok, Wang Hui, "Cross-layer design for energy efficient communication in wireless sensor networks. Wireless Communications and Mobile Computing," vol.9, pp.251-268, 2009.

[4] James M. Gilbert, Farooq Balouchi, "Comparison of energy harvesting systems for Wireless Sensor Networks," International Journal of Automation and Computing," vol.5, pp. 334-347, 2008.

[5] Arisha K A, Youssef M A, Younis M F, "Energy-aware TDMA-based MAC for sensor networks," In: Proceedings of IEEE Workshop on Integrated Management of Power Aware Communications, Computing and Networking. New York, USA: IEEE, pp.189-201, 2002.

[6] Dam TV. Langendoen K, "An adaptive energy-efficient MAC protocol for wireless sensor networks," In: Proceedings of the 1st International Conference Oll Embedded Networked Sensor Systems. California, USA: ACM, pp.171-180, 2003.

[7] Schurgers C, Tsiatsis V, Ganeriwal S, Srivastava M, "Optimizing sensor networks in the energy-latency density design space," IEEE Transactions on Mobile Computing, vol.1,2002,pp.70-80,2002.

[8] Hnin Yu Shwe, JIANG Xiaohong, Suslginu Horiguchi, "Energy saving in wireless sensor networks," Journal of Communication and Computer, vol.6, pp. 20-27, 2009.

[9] Rubin I, De Moraes L F, "Message Delay Analysis for Polling and Token Multiple-Access Schemes for Local Communication Networks," IEEE J. Select. Areas Commun, pp.935-947, 1983.

[10] 10. Abinash Mahapatra, Kumar Anand, Dharma P. Agrawal, "QoS and Energy Aware Routing for Real-Time Traffic in Wireless Sensor Networks," Computer Communications,pp.437-445, 2006.

[11] Ian F. Akyildiz, Ismail H. Kasimoglu. Wireless Sensor and Actor Networks: Research Challenges," Ad Hoc Networks, vol.2, pp.351-367, 2004. 